\title{
INFLUÊNCIA DO LOCAL DE ANCORAGEM DOS IMPLANTES NA VÉRTEBRA SOBRE O TORQUE DE INSERÇÃO E RESISTÊNCIA AO ARRANCAMENTO
}

\author{
INFLUENCE OF ANATOMICAL VERTEBRAL SITE ON SCREW INSERTION TORQUE AND \\ PULL-OUT STRENGTH
}

\author{
INFLUENCIA DE LA UBICACIÓN DE LOS IMPLANTES EN LA VÉRTEBRA CUANTO AL \\ TORQUE DE INSERCIÓN Y RESISTENCIA DE ARRANCAMIENTO
}

Rodrigo César Rosa', Patrícia Silva', Maurício José Falcal ${ }^{1}$, Antônio Carlos Shimano² ${ }^{2}$ Helton Luiz Aparecido Defino²

\begin{abstract}
RESUMO
Objetivo: Avaliar a influência do sítio anatômico da ancoragem dos implantes na vértebra sobre a resistência ao arrancamento e o torque de inserção dos parafusos pediculares com alma cônica e cilíndrica. Métodos: Parafusos cilíndricos e com alma cônica foram inseridos no pedículo e corpo vertebral de 10 vértebras lombares (L4-L5) de vitelos. Foram avaliados o torque de inserção e a resistência ao arrancamento dos parafusos inseridos no corpo e no pedículo vertebral. Resultados: Os valores do torque de inserção e resistência ao arrancamento foram maiores nos parafusos de alma cilíndrica e alma cônica inseridos no pedículo vertebral. Conclusões: A ancoragem dos implantes no pedículo vertebral apresentou maiores valores do torque de inserção e da força de arrancamento que os implantes inseridos no corpo vertebral nos dois tipos de parafusos utilizados.
\end{abstract}

Descritores: Coluna vertebral/cirurgia; Parafusos ósseos; Biomecânica; Torque.

\begin{abstract}
Objective: To evaluate the influence of anatomical site of implants on vertebra about insertion torque and pull-out strength of conical and cylindrical core screws. Methods: Conical and cylindrical core screws were inserted into the pedicle and vertebral body of 10 lumbar vertebrae (L4-L5) of calves. In the study were evaluated insertion torque and pull-out strength of screws inserted into the body and pedicle. Results: The insertion torque and pull-out strength were higher for conical and cylindrical core screws inserted into vertebral pedicle. Conclusions: The anchoring of implants into the pedicle showed higher insertion torque and pull-out strength than implants inserted into the vertebral body in both types of screws used.
\end{abstract}

Keywords: Spine; Bone screws; Biomechanics; Torque.

\section{RESUMEN}

Objetivo: Evaluar la influencia de la localización anatómica de la fijación de los implantes en la vértebra cuanto al torque de inserción y resistencia de arrancamiento de los tornillos con alma cónica y cilíndrica. Métodos: Los tornillos con alma cilíndrica y cónica se insertan en el cuerpo y el pedículo vertebral de diez vértebras lumbares (L4-L5) de los terneros. Se evaluó el torque de inserción y resistencia al arrancamiento de tornillos insertados en el cuerpo y el pedículo. Resultados: El torque de inserción y resistencia al arrancamiento fue mayor para los tornillos con alma cónica y cilíndrica insertados en el pedículo. Conclusiones: La fijación de los implantes en el pedículo mostró mayor torque de inserción y resistencia al arrancamiento en comparación con los implantes en el cuerpo vertebral, en ambos tipos de tornillos.

Descriptores: Columna vertebral; Tornillos óseos; Biomecánica; Torque.

\section{INTRODUÇÃO}

Os parafusos são componentes de ancoragem do sistema de fixação vertebral e tem sido amplamente utilizados no tratamento cirúrgico de diferentes doenças da coluna vertebral. Nas fixações anteriores esses implantes são inseridos no corpo vertebral, e o pedículo vertebral é o local de ancoragem para as fixações posteriores ${ }^{1-4}$.

As propriedades biomecânicas desses implantes e a influência de parâmetros que podem exercer influência sobre essas proprie- dades, como a densidade mineral do tecido ósseo, o preparo do orifício piloto e o desenho dos implantes tem sido relatadas ${ }^{2,5}$. No entanto, não tem sido enfatizada a importância do local de ancoragem desses implantes (corpo ou pedículo vertebral) sobre as suas propriedades biomecânicas. Os parafusos do sistema de fixação vertebral têm sido desenvolvidos para serem utilizados no corpo ou no pedículo vertebral, permitindo maior versatilidade aos sistemas de fixação. A diferença entre o corpo e o pedículo vertebral com 
relação à distribuição do osso esponjoso e a organização do osso cortical ao seu redor não tem sido apontados como fatores que poderiam interferir na resistência ao arrancamento ou torque de inserção desses implantes.

A hipótese avaliada no estudo é relacionada com a influência que a organização do tecido ósseo do corpo ou pedículo vertebral pode apresentar sobre o parafuso inserido no seu interior. Foi avaliada a hipótese de que o torque de inserção e a resistência ao arrancamento do mesmo tipo de implante colocado na mesma vértebra poderia ser diferente devido ao tipo de organização e distribuição do tecido ósseo no interior da vértebra, considerando-se o corpo e o pedículo vertebral como os locais de inserção dos implantes.

\section{MATERIAL E MÉTODO}

Foram utilizados dez segmentos de vértebras lombares (L4-L5), pertencentes a vitelos do sexo masculino, da raça holandesa, com idade de seis a oito semanas e massa corpórea média de 50,9 \pm $5,0 \mathrm{Kg}$. Os segmentos vertebrais foram armazenados em freezer à temperatura de $-20^{\circ} \mathrm{C}$. Precedendo a realização dos ensaios as vértebras foram retiradas do freezer e mantidas por 12 horas à temperatura de $5^{\circ} \mathrm{C}$, e, subsequente por duas horas à temperatura ambiente para se atingir o equilíbrio térmico e não alterar as propriedades físicas do osso. A densidade mineral óssea das vértebras foi avaliada por meio de dual x-ray absorptiometry, e a média dos valores da densidade mineral óssea foi de 0,16 $\pm 0.03 \mathrm{~g} / \mathrm{cm}^{3}$.

Foram utilizados 10 parafusos cilíndricos e 10 parafusos com alma cônica do sistema USS (Universal Spine System-Synthes) de fixação vertebral $\left(\right.$ Synthes $\left.{ }^{\circledR}\right)$, com $30 \mathrm{~mm}$ de comprimento e diâmetro externo de 5,0 $\mathrm{mm}$ (parafuso cilíndrico) e 5,2 $\mathrm{mm}$ (parafuso de alma cônica) (Figura 1).

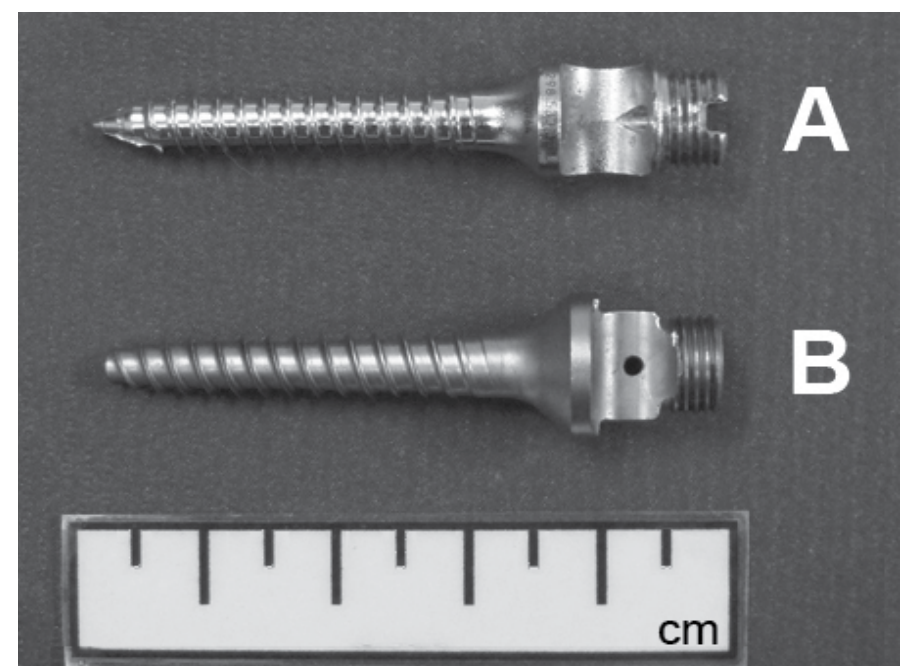

Figura 1. Parafusos utilizados no estudo. A) Parafuso Cilíndrico; B) Parafuso com Alma Cônica.

O orifício piloto foi preparado com sonda de 3,8mm de diâmetro, de acordo com a recomendação do fabricante. Os parafusos foram inseridos sob visão direta no corpo e no pedículo vertebral e os grupos experimentais formados de acordo com o tipo do parafuso utilizado (alma cilíndrica ou alma cônica). Em cada vértebra foi utilizado o mesmo tipo de parafuso para que a comparação fosse realizada em condições homogêneas.

O torque de inserção dos parafusos foi avaliado por meio de torquímetro com capacidade de $10 \mathrm{Nm}$ e Software MK versão
1.0.0.6/2004 tendo sido considerado o maior valor do torque obtido durante a inserção do implante (Figura 2).

Os ensaios mecânicos de arrancamento foram realizados utilizando máquina universal de ensaio Emicâ, Software Tesc 3.13 para

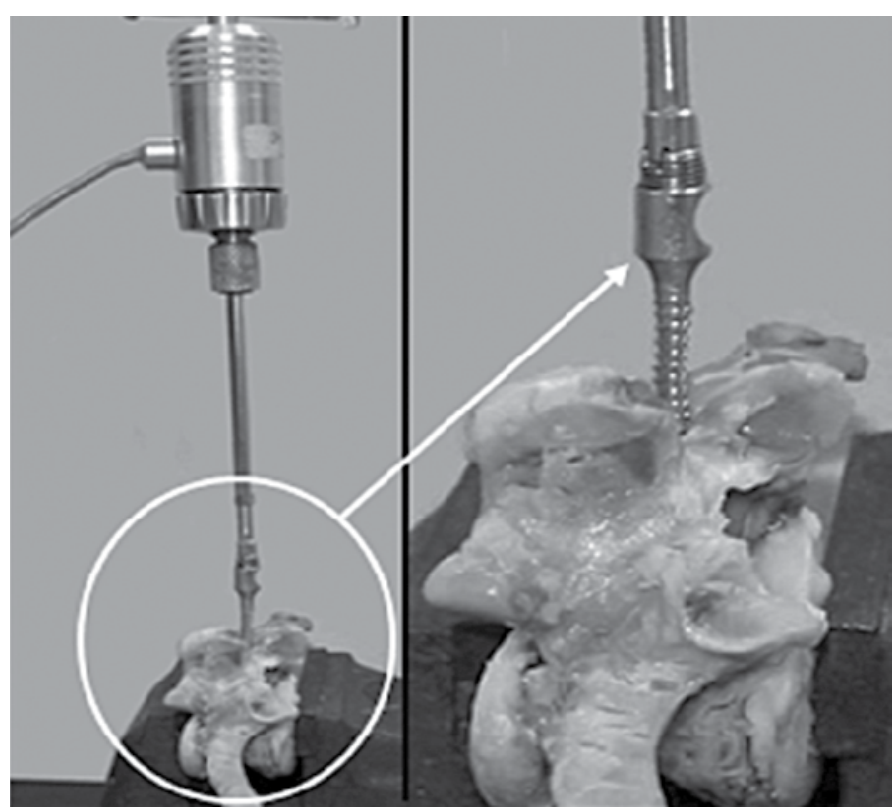

Figura 2. Inserção do implante no pedículo vertebral e a mensuração do torque de inserção.

análise dos resultados, célula de carga com capacidade de 2000 $\mathrm{N}$ e velocidade de aplicação de força de $2 \mathrm{~mm} / \mathrm{min}$. Em todos os ensaios mecânicos foi utilizada a pré-carga de 50N e tempo de acomodação de 10 segundos (Figura 3). A propriedade avaliada nos ensaios mecânicos foi à força máxima de arrancamento.

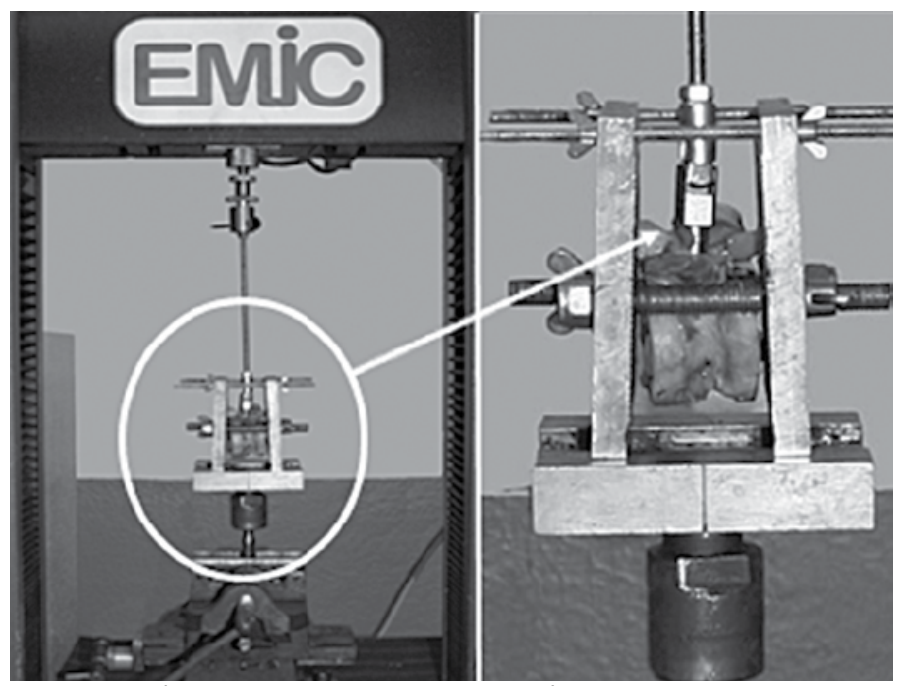

Figura 3. Máquina universal de ensaio mecânico utilizada nos testes de resistência ao arrancamento dos implantes.

Os resultados foram submetidos à análise estatística de normalidade para a determinação do comportamento da amostra (ANOVA). O nível de significância do torque de inserção e da força de arrancamento foi determinado pelo teste de Tukey, adotando nível de significância de $5 \%(p<0,05)$. 


\section{RESULTADOS}

Os resultados do torque de inserção e da força de arrancamento dos implantes estão apresentados na Tabela 1 e nas Figuras 4 e 5.

Foi observada diferença estatística entre o torque de inserção e a resistência ao arrancamento dos implantes colocados no corpo e

Tabela 1. Média dos valores do torque de inserção e força de arrancamento dos parafusos pediculares.

\begin{tabular}{c|c|c|c|c}
\hline \multirow{2}{*}{ Parafuso } & \multicolumn{2}{|c|}{ Pedículo Vertebral } & \multicolumn{2}{c}{ Corpo Vertebral } \\
\cline { 2 - 5 } & $\begin{array}{c}\text { Torque } \\
\text { (Nm) }\end{array}$ & Força (N) & $\begin{array}{c}\text { Torque } \\
\text { (Nm) }\end{array}$ & Força (N) \\
\hline $\begin{array}{c}\text { Alma } \\
\text { Cônica }\end{array}$ & $\begin{array}{c}2,63 \pm \\
0,32\end{array}$ & $1464,23 \pm 154,62$ & $2,2 \pm 0,32$ & $806,35 \pm 256,34$ \\
\hline Cilíndrico & $1,92 \pm$ & $1216,67 \pm 192,50$ & $\begin{array}{c}1,59 \pm \\
0,20\end{array}$ & $671,96 \pm 201,30$ \\
\hline
\end{tabular}

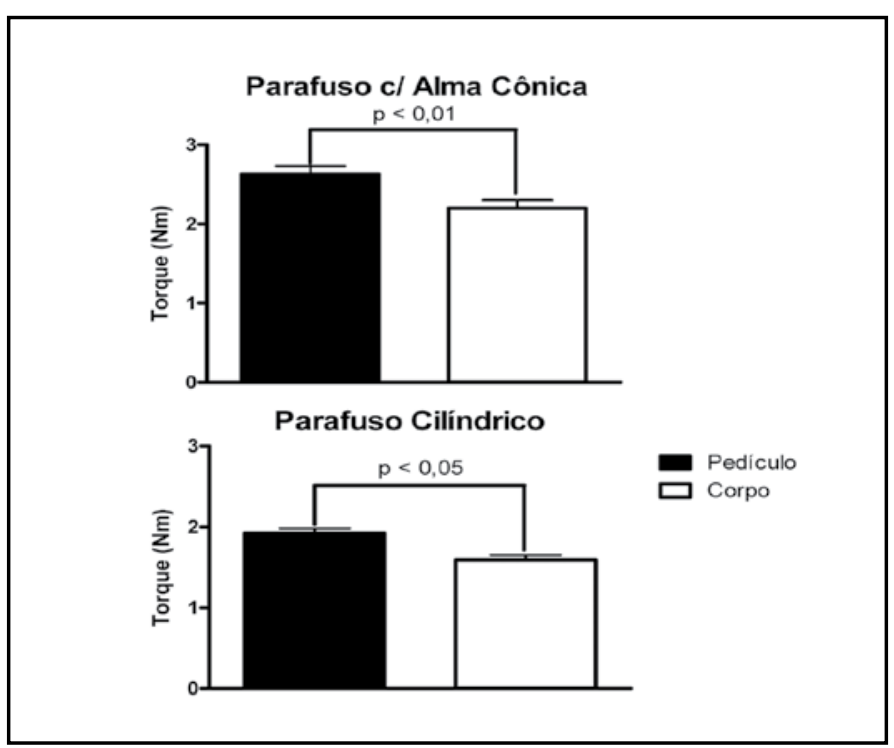

Figura 4. Torque de inserção dos parafusos pediculares.
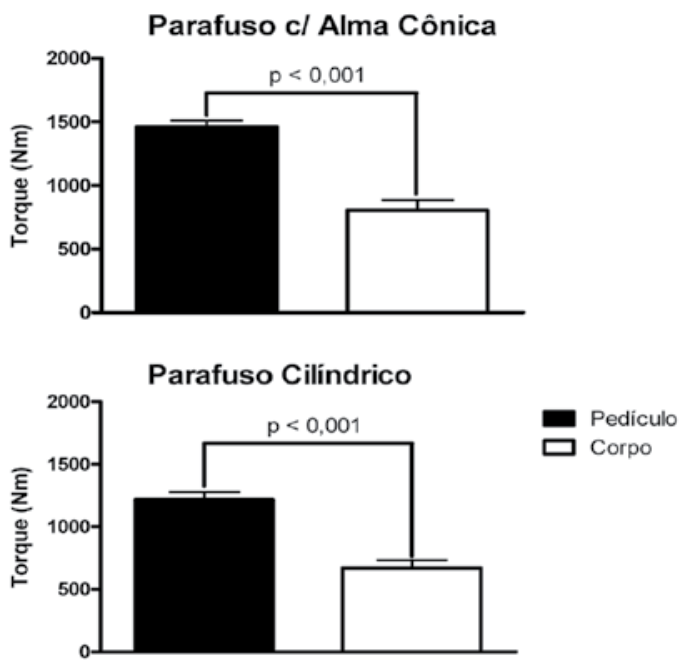

Figura 5. Força de arrancamento dos parafusos pediculares. no pedículo vertebral. Ambos os tipos de parafusos (alma cilíndrica e alma cônica) apresentaram maior torque de inserção e resistência ao arrancamento após a inserção no corpo vertebral.

\section{DISCUSSÃO}

A vértebra é basicamente constituída por osso do tipo esponjoso circundado por uma delgada camada de osso cortical em toda sua superfície externa. O corpo vertebral apresenta abundante quantidade de osso esponjoso, enquanto que nos pedículos vertebrais a quantidade de osso esponjoso é mais escassa e existe revestimento de osso esponjoso ao seu redor ${ }^{6}$. Nas situações em que o pedículo vertebral é muito delgado ou quando o diâmetro do implante colocado no seu interior excede 75 a 80\% do diâmetro interno do pedículo vertebral, pode ocorrer lesão do osso cortical do pedículo vertebral ${ }^{7,8}$. No entanto, o comportamento do osso cortical do pedículo vertebral durante a inserção de um parafuso de tamanho padrão e centrado no seu interior, ainda é desconhecido. Não está bem estabelecido que a deformação do osso cortical das paredes do pedículo vertebral poderia ocorrer pelo efeito do volume causado pela inserção do parafuso no interior do pedículo sem o contato com o osso cortical da parede do pedículo ${ }^{9}$.

Em nosso estudo o diâmetro do parafuso utilizado era inferior ao diâmetro interno do pedículo vertebral e teoricamente não ocorreu o contato do parafuso com o osso cortical da parede do pedículo devido à desproporção entre o tamanho do implante utilizado e as dimensões do pedículo vertebral.

Tem sido relatado que a deformação plástica do osso cortical do pedículo ocorre ainda que não exista contato do implante com o osso cortical da parede do pedículo ${ }^{8}$.

A maior resistência ao arrancamento e o maior torque de inserção dos parafusos inseridos no pedículo vertebral pode estar relacionada com a impacção do osso esponjoso sobre o osso cortical da parede do pedículo ao seu redor ${ }^{7}$. Tem sido relatado que a densidade do osso esponjoso no interior do pedículo não corresponde à observada na densitometria óssea do corpo da vértebra $^{12}$. A menor resistência ao arrancamento dos parafusos inseridos no corpo vertebral tem sido observada nos pacientes submetidos à correção de escoliose por via anterior, especialmente na coluna torácica ${ }^{11-17}$.

O torque e a resistência ao arrancamento dos implantes aplicados na vértebra são influenciados pela densidade mineral do osso, desenho do implante, orifício piloto, e também pelo local anatômico da vértebra na qual o implante é colocado. A diferença de organização do osso esponjoso do corpo vertebral em relação ao pedículo vertebral, e a existência de osso cortical ao seu redor, influenciam as propriedades mecânicas dos implantes colocados no seu interior.

\section{CONCLUSÃO}

O sítio anatômico (corpo ou pedículo vertebral) influência no torque de inserção e na resistência ao arrancamento dos parafusos cilíndricos ou com alma cônica inseridos na vértebra.

\section{AGRADECIMENTOS}

Agradecemos ao apoio que nos foi dado pela FAPESP (Processo No 2007/03712-0) na realização desse trabalho. 


\section{REFERÊNCIAS}

1. Carmouche JJ, Molinari RW, Gerlinger T, Devine J, Patience T. Effects of pilot hole preparation technique on pedicle screw fixation in different regions of the osteoporotic thoracic and lumbar spine. J Neurosurg Spine. 2005;3(5):364-70.

2. Chen PQ, Lin SJ, Wu SS, So H. Mechanical performance of the new posterior spinal implant: effect of materials, connecting plate, and pedicle screw design. Spine (Phila Pa 1976). 2003;28(9):881-6.

3. Coe JD, Warden KE, Herzig MA, McAfee PC. Influence of bone mineral density on the fixation of thoracolumbar implants. A comparative study of transpedicular screws, laminar hooks, and spinous process wires. Spine (Phila Pa 1976). 1990;15(9):902-7.

4. Hsu CC, Chao CK, Wang JL, Hou SM, TsaiYT, Lin J. Increase of pullout strength of spinal pedicle screws with conical core: biomechanical tests and finite element analyses. J Orthop Res. 2005;23(4):788-94.

5. Kuhn A, Mc IffT, Cordey J, Baumgart FW, Rahn B A. Bone deformation by thread-cutting and thread- forming cortex screws. Injury. 1995;26(Suppl 1):S-A12-20.

6. Inceoğlu S, Kilinçer C, Tami A, McLain RF. Cortex of the pedicle of the vertebral arch. Part I: Deformation characteristics during screw insertion. J Neurosurg Spine. 2007;7(3):341-6.

7. Defino HL, Vendrame JR. Role of cortical and cancellous bone of the vertebral pedicle in implant fixation. Eur Spine J. 2001;10(4):325-33.

8. Misenhimer GR, Peek RD, Wiltse LL, Rothman SL, Widell EH Jr. Anatomic analysis of pedicle cortical and cancellous diameter as related to screw size. Spine (Phila Pa 1976). 1989;14(4):367-72.
9. Abshire BB, McLain RF, Valdevit A, Kambic HE. Characteristics of pullout failure in conical and cylindrical pedicle screws after full insertion and back-out. Spine J. 2001;1(6):408-14.

10. Inceoglu S, Burghardt A, Akbay A, Majumdar S, McLain RF. Trabecular architecture of lumbar vertebral pedicle. Spine (Phila Pa 1976). 2005;30(13):1485-90.

11. Alobaid A, Arlet $V$, Busato $A$, Steffen T. Pull-out strength of the suprapedicle claw construct: a biomechanical study. Eur Spine J. 2005;14(8):759-64.

12. Breeze SW, Doherty BJ, Noble PS, LeBlanc A, Heggeness MH. A biomechanical study of anterior thoracolumbar screw fixation. Spine (Phila Pa 1976). 1998;23(17):1829-31.

13. Chapman JR, Harrington RM, Lee KM, Anderson PA, Tencer AF, Kowalski D. Factors affecting the pullout strength of cancellous bone screws. J Biomech Eng. 1996;118(3):391-8.

14. DeCoster TA, Heetderks DB, Downey DJ, Ferries JS, Jones W. Optimizing bone screw pullout force. J Orthop Trauma. 1990;4(2):169-74.

15. Horton WC, Blackstock SF, Norman JT, Hill CS, Feiertag MA, Hutton WC. Strength of fixation of anterior vertebral body screws. Spine (Phila Pa 1976).1996;21(4):439-44.

16. Lenke LG. Anterior endoscopic discectomy and fusion for adolescent idiopathic scoliosis. Spine (Phila Pa 1976). 2003;28(15 Suppl):S36-43.

17. Lieberman $\mathrm{IH}$, Khazim $\mathrm{R}$, Woodside T. Anterior vertebral body screw pullout testing. A comparison of Zeilke, Kaneda, Universal Spine System, and Universal Spine System with pullout-resistant nut. Spine (Phila Pa 1976). 1998;23(8):908-10. 